Article

\title{
Quantitative Mereology: An essay to derive physics laws from a philosophical concept
}

\author{
Georg J. Schmitz \\ ACCESS e.V., Intzestr.5, D-52072 Aachen, Germany; \\ G.J.Schmitz@access.rwth-aachen.de; Tel.: +49-241-80-98014 \\ Received: date; Accepted: date; Published: date
}

\begin{abstract}
Mereology stands for the philosophical concept of parthood and is based on a sound set of fundamental axioms and relations. One of these axioms relates to the

existence of a universe as a thing having part all other things.
\end{abstract}

The present article formulates this logical expression first as an algebraic inequality and eventually as an algebraic equation reading in words:

The universe equals the sum of all things.

"All things" here are quantified by a "number of things". Eventually this algebraic equation is normalized leading to an expression

The whole equals the sum of all fractions.

This introduces " 1 " or " $100 \%$ " as a quantitative - numerical - value describing the "whole". The resulting "basic equation" can then be subjected to a number of algebraic operations. Especially squaring this equation leads to correlation terms between the things implying that the whole is more than just the sum of its parts. Multiplying the basic equation (or its square) by a scalar allows for the derivation of physics equations like the entropy equation, the ideal gas equation, an equation for the Lorentz-Factor, conservation laws for mass and energy, the energy-mass equivalence, the Boltzmann statistics, and the energy levels in a Hydrogen atom. It further allows deriving a "contrast equation" which may form the basis for the definition of a length and a time scale. Multiplying the basic equation with vectors, pseudovectors, pseudoscalars and eventually hypercomplex numbers opens up the realm of possibilities to generate many further equations.

Keywords: entropy; Boltzmann statistics; Lorentz-factor; mereology; analytical philosophy; conservation laws; vacuum energy; cosmologic constant problem; invariance; hypercomplex numbers; Octonions; energy-mass equivalence; Ur-Alternatives; mereotopology; mereophysics

\section{Introduction}

Epistemology and Gnosiology are major areas of philosophy addressing questions related to pre-conditions for the acquisition of insights and discoveries, the generation and accumulation of knowledge and to origins of other forms of perception and beliefs [adapted from [1]]. Gnosiology ("study of knowledge") is "the philosophy of knowledge and cognition"[2]. "... as a philosophical concept, gnosiology broadly means the theory of knowledge, which in ancient Greek philosophy was perceived as a combination of sensory perception and intellect and then made into 
memory..."[3]. The term gnosiology in this reference is then further elaborated for the area of science: "...in the context of science, gnosiology takes on a different meaning: the study of knowledge, its origin, processes, and validity".

Both - epistemology and gnosiology - draw on the definition of words and their meanings being at the core of ontology for which following specification is given (cited from [4]). "Ontology is the philosophical study of the nature of being, becoming, existence, or reality, as well as the basic categories of being and their relations". Traditionally listed as a part of the major branch of philosophy known as metaphysics, ontology often deals with questions concerning what entities exist or may be said to exist and how such entities may be grouped, related within a hierarchy, and subdivided according to similarities and differences [5]. A very simple definition of ontology is that it is the examination of what is meant, in context, by the word 'thing'.

Ontologies currently gain increasing importance in the area of organizing knowledge, of providing a common language, of improving interoperability between humans and computers or between different tools being performed on computers. Ontologies also find applications in artificial intelligence and machine learning approaches. The European Materials \& Modelling Ontology "EMMO" has recently been elaborated [6], [7]. This ontology is constructed from very fundamental principles and covers ontologies for both models and materials and also for semiotics i.e. the way how a meaning is assigned to symbols. The work on this ontology has catalyzed the present article.

The first version of EMMO at its uppermost, abstract level classified things into "sets" and "things, which are not a set" the latter being called "items" in EMMO. All "sets" in this ontology were subject to classical "set theory" like the ZFC (the "Zermelo-Fraenkel set theory including the axiom of choice [8]). In the actual version of EMMO, however, sets are replaced by collections having at least two members and thus not being sets because not allowing for singletons [9].

The "item" class in the EMMO in a first place was defined as being disjoint from the "set" class and thus comprises "anything which is not a set". Interestingly this definition relates to C.F. von Weizsäcker's "Ur-alternatives" being defined as "anything which is not a set" [10]. The EMMO then provides a further interpretation of "items" as fusions of things, which underlie the philosophical concepts of mereology resp. mereotopology. The major characteristic relations in mereology are the has_part resp. item is_part_of relations. Interestingly, all physical objects in EMMO are categorized as items i.e. as things being described by mereology.

The term mereology was first coined by Stanisław Leśniewski as one of three formal systems: protothetic, ontology, and mereology. "Leśniewski was also a radical nominalist: he rejected axiomatic set theory at a time when that theory was in full flower. He pointed to Russell's paradox and the like in support of his rejection, and devised his three formal systems as a concrete alternative to set theory" [11]. The mereological and mereotopological concepts like the different types of parthood relations as used the EMMO are based on the book "Parts \& Places" [12] and the references therein.

The present article is an essay to quantify mereology. The scope is - starting from first order logic expressions and axioms of mereology - to provide an algebraic equation comprising at least one numerical value. Once being available, such an algebraic equation in view of its fundamental nature may become the source to derive further equations in a deductive manner. In the following the reasoning for the individual steps towards such an equation is provided before eventually deducing some physics laws from it. 


\section{From mereology to quantitative mereology}

The term mereology originates from Ancient Greek $\mu$ ćoos (méros, "part") + -logy ("study, discussion, science") while the term topology from Ancient Greek tó -(o)logy ("study of, a branch of knowledge"). The combined expression mereotopology stands for a theory combining mereology and topology and the philosophical branch of mereotopology aims at investigating relations between parts and wholes and boundaries between them.

The fundamentals of mereology and mereotopology are described in full detail with all logical expressions, axioms and their derivation from First Order Logic in [13] and are not detailed here. One of the axioms of mereology relates to the existence of a universe as an item which has part all other items [12],[14]:

Axiom ("Top"): There exists a "universal object", designated W, such that PxW (read "x is part of $\left.W^{\prime \prime}\right)$ holds for any $x$, meaning that all $x$ are part of $W$ :

$$
\exists \mathrm{W} \forall \mathrm{x}[\mathrm{PxW}]
$$

In the following and throughout the entire article a "quantification" of this logical expression is attempted. The first step towards this quantification relates to the "all quantifier" $\forall$. "All" in the present article in a first step is quantified as the number $N_{x}$ of things $x_{i}\left(i=1, . ., N_{x}\right)$ constituting the present universe. Reasoning for the further extension to "All" is provided in the course of this article.

The universe $\mathrm{W}$ existing according to above mereological axiom has part all things and accordingly must be greater or equal than the sum of the things:

$$
W \geq \sum_{i=1}^{N_{x}} x_{i}
$$

It follows that there exists an $x_{0}$ - a matrix thing - which - when being added - generates equality:

$$
W=x_{0}+\sum_{i=1}^{N_{x}} x_{i}=\sum_{i=0}^{N_{x}} x_{i}
$$

This sum additionally includes the "matrix" thing $x_{0}$, which has part all other things, Fig 1.

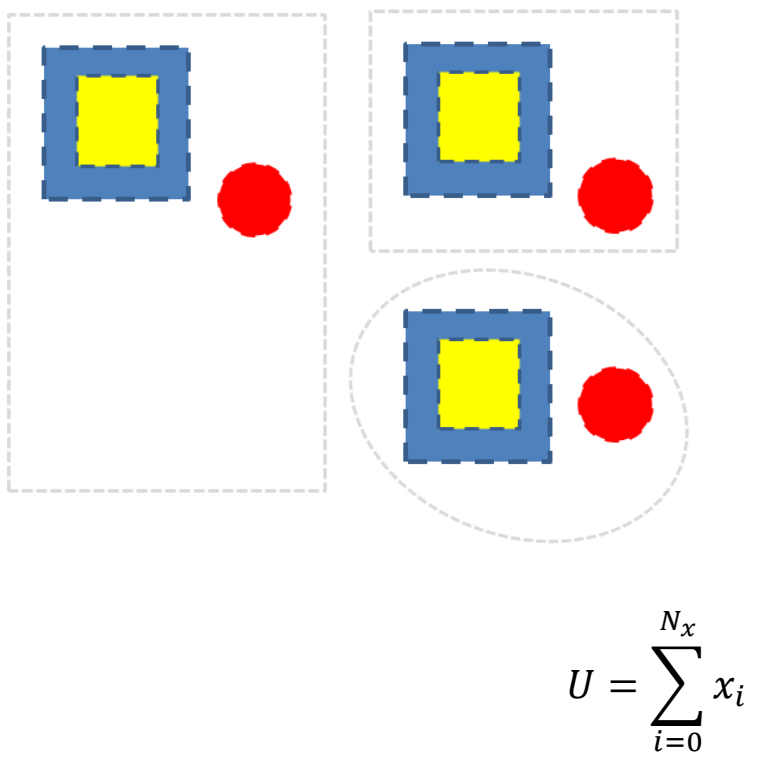

Fig 1: Different "matrix" things $x_{0}$ (marked by gray dashed boundaries) enclosing the same set of things (blue yellow, red). They all have_part all other things and thus represent possible universes according to the mereology axiom. The relative size and shape of the $x_{0}$ domains may differ. Selecting one of the $x_{0}$ fixes the universe $U$ under consideration. 
The universe $U$ according to this expression is the sum of its parts including the matrix. In a further quantification step one can "normalize" this universe getting the numerically quantified value " 1 ":

$$
1=\sum_{i=0}^{N_{x}} \frac{x_{i}}{U}
$$

Renaming the $x_{i} / U$ - being the fractions of the universe - to $\Phi \mathrm{i}$ leads to the "basic equation":

$$
1=\sum_{i=0}^{N_{\Phi}} \Phi_{i}
$$

\section{(basic equation)}

This equation expressed in words reads:

\section{The "whole" is the sum of its fractions \\ (including the matrix as a fraction)}

Several assumptions have been made above to motivate this formula on the basis of a mere logical expression. While a strong need for a rigorous derivation arises, quite a number of important conclusions can be drawn from further investigating this formula in detail. In the following all "things" are interpreted as being fractions of the universe.

The universe, the whole, or just everything - named " 1 " - is the sum of all (a very large but countable number) fractions - whether they are existing or they are not-existing. The name of each thing is $\Phi_{i}$ with the index i (a positive integer number) being used to distinguish a thing i from another thing $\mathrm{j}$.

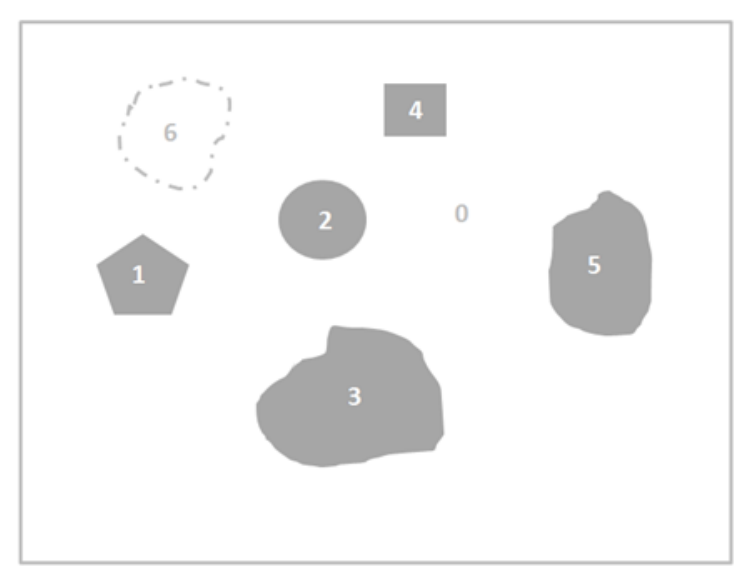

Fig: 2: A universe of things embedded in a spacetime region (limited by the frame). All of the things have boundaries with the vacuum/matrix " 0 " only. Thing 7 is not considered to be a part of this particular universe/ spacetime-region. Thing 6 is a thing which might have been part of the universe in the past or might become part of the universe in the future, but does not exist in the considered time-interval. The total number of existing things in this region $N_{\Phi}$ thus is 5 and together with the matrix $\Phi_{0}$ sums up to 6 .

An existant denotes a thing existing in a finite period of time. This comprises all things/objects, which exist in a system during an observation. The system may be defined as a region under consideration or even as the entire universe. The number of existants is called $N_{\Phi}$ and may be very large. All existants sum up to the maximum of 1 (meaning 100\% of all known (or considered) 
existing things). In absence of any other thing (i.e. for $N_{\Phi}=0$ ), the vacuum $\Phi_{0}$ remains as the only existant.

The basic equation - which has already unveiled its power in multi-phase-fields models [15] describing the evolution of complex structures [16] - holds everywhere and any time i.e.:

$$
\sum_{i=0}^{N_{\Phi}} \Phi_{i}(x, t)=1 \text { for all } x \text { and all } t
$$

If a thing is "existent" it takes a non-zero value [17]:

$$
0<\Phi_{i} \leq 1
$$

If a thing is "non-existent" (or "not yet existent" or "no more existent") it takes exactly the value zero with the potential of changing to a non-zero value i.e. with the potential of becoming an existent thing. The all quantifier $\forall$ in the original mereological expression can in this way de-facto be recovered by extending the sum from minus to plus infinity yielding the fundamental equation:

$$
\begin{gathered}
\sum_{-\infty}^{\infty} \Phi_{i}=\sum_{-\infty}^{-1} \Phi_{i}+\sum_{i=0}^{N_{\Phi}} \Phi_{i}+\sum_{i=N_{\Phi^{+}}}^{\infty} \Phi_{i}=1 \\
\sum_{-\infty}^{\infty} \Phi_{i}=\text { past things }+ \text { present things }+ \text { future things }=1
\end{gathered}
$$

\section{(fundamental equation)}

Because past \& future things are "not more" or "not yet" existing they all take exactly the value zero and the fundamental equation thus reduces to the basic equation:

$$
\sum_{-\infty}^{\infty} \Phi_{i}=\sum_{i=0}^{N_{\Phi}} \Phi_{i}=1
$$

Note that the number of things $N_{\Phi}$ not necessarily is a constant. New things may occur by splitting of existing things (increasing the number $N_{\Phi}$ ) or new things may be created by fusion of existing things (decreasing the number $N_{\Phi}$ ).

If a thing is the only thing existing in a region, it takes the value 1 in that particular region of spacetime. The thing " 0 " can be considered as the matrix, as "the vacuum", or simply as a region of "spacetime" itself.

It can be noted that this basic equation is a scalar equation and does not contain any information neither about time nor about space. It thus is independent of space and time and holds anywhere and anytime. Accordingly, it is also invariant under translations and rotations in space and also invariant under translations in time. Following the Noether theorem these invariances directly imply the conservation of quantities like momentum, spin and energy [18]. The equation also accounts for the requirements of unitarity and locality being fundamental principles of quantum mechanics. The sequence of summing up things does not change the result. This corresponds to the commutative law in mathematics and is important as it allows for sorting of things and correlations between neighbored things.

\section{Correlations}


The whole is more than the sum of its fractions

Things may interact with other things. Such interactions correspond to correlations between the things. These correlations only exist if the things coexist during a finite interval of time. Things coexist at least at their mutual boundary.

The basic equation can easily be squared:

$$
\sum_{i=0}^{N_{\Phi}} \Phi_{i}=1 \rightarrow\left(\sum_{i=0}^{N_{\Phi}} \Phi_{i}\right)^{2}=1^{2}=1
$$

which provides:

$$
\left(\sum_{i=0}^{N_{\Phi}} \Phi_{i}\right)^{2}=1^{2}=1=\Phi_{i}^{2}+\Phi_{j}^{2}+\cdots+\Phi_{i} \Phi_{j}+\Phi_{j} \Phi_{i}+\cdots+\cdots=\sum_{i=0}^{N_{\Phi}} \Phi_{i}^{2}+\sum_{\substack{i, j=0 \\ i \neq j}}^{N_{\Phi}} \Phi_{i} \Phi_{j}
$$

In short this reads:

$$
1=\sum_{i=0}^{N_{\Phi}} \Phi_{i}{ }^{2}+\sum_{\substack{i, j=0 \\ i \neq j}}^{N_{\Phi}} \Phi_{i} \Phi_{j}
$$

This procedure obviously introduces correlation terms of type $\Phi_{i} \Phi_{j}$ between different things, i.e. terms describing the coexistence resp. interactions of pairs of things. Such terms are only present if the things are coexistent i.e. both things have non-zero values. These terms can be further elucidated as follows. This equation in combination with the basic equation (equation (7), repeated here for better readability)

$$
\sum_{i=0}^{N_{\Phi}} \Phi_{i}=1
$$

gives

$$
-\sum_{\substack{i, j=0 \\ i \neq j}}^{N_{\Phi}} \Phi_{i} \Phi_{j}=\sum_{i=0}^{N_{\Phi}} \Phi_{i}^{2}-1=\sum_{i=0}^{N_{\Phi}} \Phi_{i}^{2}-\sum_{i=0}^{N_{\Phi}} \Phi_{i}=\sum_{i=0}^{N_{\Phi}}\left(\Phi_{i}^{2}-\Phi_{i}\right)
$$

The term in brackets on the right hand side of this equation interestingly corresponds to the lowest order of the Taylor expansion of an entropy type logarithmic formulation [19]:

$$
\begin{gathered}
\Phi_{i}^{2}-\Phi_{i} \cong \Phi_{i} \ln \Phi_{i} \quad\left(\text { for } \Phi_{i} \leq 1\right) \\
\sum_{i=0}^{N_{\Phi}} \Phi_{i} \ln \Phi_{i} \cong \sum_{i=0}^{N_{\Phi}}\left(\Phi_{i}^{2}-\Phi_{i}\right)
\end{gathered}
$$

In total, the correlations of one thing with all other things can thus obviously be expressed by a function of that single thing only: 


$$
\begin{gathered}
\sum_{\substack{i, j=0 \\
i \neq j}}^{N_{\Phi}} \Phi_{i} \Phi_{j} \cong-\sum_{i=0}^{N_{\Phi}} \Phi_{i} \ln \Phi_{i} \quad \text { ("correlation equation") } \\
S=-\sum_{i=0}^{N_{\Phi}} \Phi_{i} \ln \Phi_{i} \\
\text { ("entropy equation)" }
\end{gathered}
$$

This term $S$ is the well-known entropy formulation appearing in Gibbs's thermodynamics ([20], [21]), in the derivation of Boltzmann's statistics [22], or in Shannon's theory of communication [23]. This seems a very important intermediate result:

\section{entropy type terms are related to correlations of things}

Because correlations exist at least at interfaces - entropy type terms are related to interfaces. As a side remark, also the Bekenstein-Hawking entropy of a black hole in its dimensionless notation $S_{B H}=\frac{A}{4 l_{p}^{2}}$ is proportional to the area of the event horizon, which is an interface [24].

Correlations further correspond to interactions and eventually interactions between things correspond to forces. Forces are often described as gradients of scalar fields. Such gradients exist at boundaries between things. Correlations have further been shown to be related to entropy type terms. The terms correlation, interaction, force, gradient, entropy, and boundary for this reason seem to be strongly interrelated and may even be based on a common principle like quantitative mereotopology [25].

\section{Deriving physics laws}

An important ingredient in the basic equation is the total number of things $N_{\Phi}$. This total number of things can be exploited to derive some quite fundamental equations of physics as will be shown in the following sections.

\subsection{Conservation laws}

A property E (let it be the property "energy") can be assigned by multiplying the basic equation with a scalar E having - or not having - also a unit:

$$
\begin{gathered}
\sum_{i=0}^{N_{\Phi}} \Phi_{i} E=E \\
\sum_{i=0}^{N_{\Phi}} E_{i}=E \\
\sum_{i=0}^{N_{\Phi}} \frac{E_{i}}{E}=1
\end{gathered}
$$

In words: the sum of all energies is a positive constant. The time derivative of this equation reads 


$$
\sum_{\substack{\mathrm{i}=0 \\ \text { (energy conservation) }}}^{\mathrm{N}_{\Phi}} \dot{\mathrm{E}}_{\mathrm{i}}=0
$$

which allows exchanging energy between the different states but makes the property $\mathrm{E}$ an overall conserved quantity. In a similar way, we can assign another property $M$ (let it be the property "mass")

$$
\begin{gathered}
\sum_{i=0}^{N_{\Phi}} \Phi_{i} M=M \\
\sum_{i=0}^{N_{\Phi}} M_{i}=M \\
\sum_{i=0}^{N_{\Phi}} \frac{M_{i}}{M}=1
\end{gathered}
$$

In words: the sum of all masses is a constant. The time derivative of this equation reads

$$
\sum_{i=0}^{N_{\Phi}} \dot{M}_{i}=0
$$

\section{(mass conservation)}

which allows exchanging mass between the different states but makes the property $M$ an overall conserved quantity.

A first conclusion, which can be drawn from the above is that any scalar value being multiplied to the basic equation would be a conserved quantity. Any scalar value can, however, be represented as a sum, as a product or as a sum of products of other scalar values.

To allow for summation of different entities having different units, a conversion of units by some conversion factors has to be assured. The conversion factor between energy and mass units is a priori unknown and may be called $\mathrm{c}^{2}$ (to account for prior knowledge/conventions)

$$
\begin{gathered}
\sum_{i=0}^{N_{\Phi}} \Phi_{i}\left(E+M c^{2}\right)=\left(E+M c^{2}\right) \\
\sum_{i=0}^{N_{\Phi}}\left(E_{i}+M_{i} c^{2}\right)=\left(E+M c^{2}\right) \\
\sum_{i=0}^{N_{\Phi}}\left(\dot{E}_{i}+\dot{M}_{i} c^{2}\right)=0 \\
\sum_{i=0}^{N_{\Phi}} \dot{E}_{i}+\sum_{i=0}^{N_{\Phi}} \dot{M}_{i} c^{2}=0
\end{gathered}
$$

This equation if fulfilled in case both entities are conserved individually. In general, however, this implies that there is only one overall conserved property - the sum of all dimensionless 
properties - which is conserved for sure. Conversion of the property mass to the property energy and vice versa is possible on the basis of this equation.

\subsection{Energy-Mass Equivalence}

Using the above equations we can further write

$$
\begin{gathered}
\sum_{i=0}^{N_{\Phi}} \frac{M_{i}}{M}=1=\sum_{i=1}^{N_{\Phi}} \frac{E_{i}}{E} \\
\sum_{i=0}^{N_{\Phi}} M_{i} \frac{E}{M}=\sum_{i=1}^{N_{\Phi}} E_{i} \\
0=\sum_{i=0}^{N_{\Phi}}\left\{E_{i}-M_{i} \frac{E}{M}\right\}
\end{gathered}
$$

In case all individual contributions are greater equal 0 it follows that each individual contribution to the sum has to be identical 0 :

$$
0=E_{i}-M_{i} \frac{E}{M}
$$

with $\mathrm{E} / \mathrm{M}$ - the ratio of the total energy of the universe and the total mass of the universe - being a universal physical constant. Naming this constant $\mathrm{c}^{2}$

$$
\frac{E}{M}=c^{2}
$$

one gets the well-known Einstein relation between mass and energy:

$$
\begin{gathered}
E_{i}=M_{i} c^{2} \\
\text { (energy }- \text { mass equivalence) }
\end{gathered}
$$

\subsection{The Lorentz-factor}

Let there be some additional type of "energy" called Ekin so that the total energy reads

$$
E=E^{0}+E^{k i n}
$$

As all energy is positive, $\mathrm{E}$ is larger than $\mathrm{E}^{0}$ by some positive factor $\gamma$ (which is greater equal 1 ) and can be written as

$$
\begin{gathered}
E=\gamma E^{0} \\
\gamma E^{0}=E^{0}+E^{k i n}
\end{gathered}
$$

Solving this equation for $\gamma$ yields

$$
\gamma=\frac{E^{0}+E^{k i n}}{E^{0}}
$$

This formula fully defines the Lorentz factor although not in its usual form [26]

4.4. Derivation of the ideal gas law 
The derivation of the ideal gas law is based on describing the averages of properties (noted as values in brackets). The average_volume of things corresponds to the total volume divided by the number of things:

$$
\sum_{i=0}^{N_{\Phi}} \Phi_{i} V=\sum_{i=0}^{N_{\Phi}} V_{i}=V=\left(N_{\Phi}+1\right)\langle V\rangle
$$

The average_energy per thing can be described in an analogue way as

$$
\sum_{i=0}^{N_{\Phi}} \Phi_{i} E=\sum_{i=0}^{N_{\Phi}} E_{i}=E=\left(N_{\Phi}+1\right)\langle E\rangle
$$

The additional " +1 " in these equations arises from extending the sum also over the vacuum/matrix thing $\Phi_{0}$, which is an inherent part of the basic equation. In case of a large number of molecules/atoms in a gas (e.g. $10^{23}$ atoms) this additional " 1 " does not play any role as in this case (with an error of 10-23):

$$
N_{\Phi}+1 \sim N_{\Phi}
$$

The average_energy per thing is the basis for the definition of the temperature $\mathrm{T}$ :

$$
\langle E\rangle=\frac{E}{N_{\Phi}} \quad(\sim k T)
$$

The average_energy per volume i.e. the average_energy_density (commonly denoted as pressure p) reads

$$
\frac{E}{V}=\frac{\langle E\rangle}{\langle V\rangle} \quad(\sim p)
$$

Equating these average energies yields

$$
p V=\frac{\langle E\rangle}{\langle V\rangle} V=\frac{\langle E\rangle}{\langle V\rangle} N_{\Phi}\langle V\rangle=N_{\Phi}\langle E\rangle=N_{\Phi} \frac{E}{N_{\Phi}}=N_{\Phi} k T
$$

which ultimately results in the ideal gas equation:

$$
\begin{gathered}
p V=N_{\Phi} k T \\
\text { (ideal gas equation) }
\end{gathered}
$$

Not neglecting the " +1 " in equation (34) leads to

$$
\begin{gathered}
p V=\left(N_{\Phi}+1\right) k T \\
\text { (modified ideal gas equation) }
\end{gathered}
$$

Interestingly this equation also relates energy to the number of states and to temperature and introduces the conversion factor $\mathrm{k}$ :

$$
E=\left(N_{\Phi}+1\right) k T
$$


In absence of all other things equation (39), however, implies the existence of a "vacuum energy"

$$
E_{0}=\langle E\rangle \text { for } N_{\Phi}=0
$$

For a single thing/state in a vacuum/matrix on gets:

$$
E_{0}+E_{1}=2\langle E\rangle \text { for } N_{\Phi}=1
$$

This case can probably be identified with the ground state of a quantum mechanical system. In any case the formula suggests that the vacuum is just one further degree of freedom in a thermodynamic system. In thermodynamic equilibrium in this two state system (ground state and vacuum) it takes a value of $1 / 2 \mathrm{kT}$. In the prior case with $10^{23}$ particles only a share of $10^{-23} \mathrm{kT}$ remains for the vacuum. However, based on this formula, it can be stated that the vacuum energy in this interpretation is a non-zero, positive and finite value. Further investigations on this interpretation might be interesting with respect to the cosmological constant problem [27], which shows discrepancies for the value of the vacuum energy of more than 120 orders of magnitude [28].

\subsection{Energy levels in the Hydrogen atom}

The average energy in a system with $n$ things/states reads

$$
\left\langle E_{n}\right\rangle=\frac{E}{n}
$$

The change of this average energy when adding one or more further states during a time interval $\mathrm{T}$ reads

$$
\left\langle E_{m}\right\rangle-\left\langle E_{n}\right\rangle=\int_{0}^{T} \frac{\partial\langle E\rangle}{\partial n} \frac{\partial n}{\partial t} d t
$$

With

and

$$
\frac{\partial\langle E\rangle}{\partial \mathrm{n}}=-\frac{\mathrm{E}}{\mathrm{n}^{2}}
$$

$$
n(t=0)=n \text { and } n(t=T)=m
$$

and assuming a "sharp" - delta function type - change rate

$$
\frac{\partial \mathrm{n}}{\partial \mathrm{t}}=\delta\left(\mathrm{t}-\mathrm{t}_{0}\right)
$$

this integral eventually yields

$$
\left\langle E_{m}\right\rangle-\left\langle E_{n}\right\rangle=E\left(\frac{1}{n^{2}}-\frac{1}{m^{2}}\right)
$$

Renaming the energy E to Ry (the so called Rydberg energy) gives

$$
\begin{gathered}
\left\langle E_{m}\right\rangle-\left\langle E_{n}\right\rangle=R_{y}\left(\frac{1}{n^{2}}-\frac{1}{m^{2}}\right) \\
\text { (energy levels in hydrogen) }
\end{gathered}
$$

which exactly corresponds to the formula for describing the energy levels in the hydrogen atom [29]. 
Using equations (14) and (16) one can write

$$
\sum_{i=0}^{N_{\Phi}} \Phi_{i} \ln \Phi_{i}-\sum_{i=0}^{N_{\Phi}} \Phi_{i}^{2}=1=\sum_{i=0}^{N_{\Phi}} \Phi_{i}
$$

Assigning the property "energy" proceeds by multiplication of both sides with E and provides

$$
E \sum_{i=0}^{N_{\Phi}} \Phi_{i} \ln \Phi_{i}-E \sum_{i=0}^{N_{\Phi}} \Phi_{i}^{2}=E \sum_{i=0}^{N_{\Phi}} \Phi_{i}
$$

with

$$
E \Phi_{i}=E_{i}\left(\text { and accordingly } E \Phi_{i}^{2}=\Phi_{i} E_{i}\right) \text { and }\langle E\rangle=\frac{E}{N_{\Phi}}
$$

this equation gives

$$
N_{\Phi}\langle E\rangle \sum_{i=0}^{N_{\Phi}} \Phi_{i} \ln \Phi_{i}-\sum_{i=0}^{N_{\Phi}} \Phi_{i} E_{i}=N_{\Phi}\langle E\rangle
$$

Renaming the average energy to

$$
N_{\Phi}\langle E\rangle=\frac{1}{\lambda}\left(\text { with } \frac{1}{\lambda} \text { to be identified with } k T\right)
$$

and multiplying both sides with $\lambda$ yields

$$
N_{\Phi}\langle E\rangle \sum_{i=0}^{N_{\Phi}} \Phi_{i} \ln \Phi_{i}-\sum_{i=0}^{N_{\Phi}} \Phi_{i} E_{i}=N_{\Phi}\langle E\rangle
$$

This ultimately leads to following dimensionless equation:

$$
\sum_{i=0}^{N_{\Phi}} \Phi_{i} \ln \Phi_{i}-\lambda \sum_{i=0}^{N_{\Phi}} \Phi_{i} E_{i}-\sum_{i=0}^{N_{\Phi}} \Phi_{i}=0
$$

This equation can directly be compared to the free energy functional in classical thermodynamics being based on Gibbs's formulation of the following variational problem, see e.g. [30]:

$$
\frac{\delta}{\delta \Phi_{i}}\left\{\sum_{i=0}^{N} \Phi_{i} \ln \Phi_{i}-\lambda\left(\sum_{i=0}^{N} \Phi_{i} E_{i}-E_{\text {tot }}\right)-\mu\left(\sum_{i=0}^{N} \Phi_{i}-1\right)\right\}=0
$$

with $\lambda$ and $\mu$ being the Lagrange multipliers accounting for the constraints of energy and probability conservation. The solution of this variational problem yields the well-known relation for the probability of specific state $\Phi_{i}$ :

$$
\Phi_{i}=e^{-\lambda \mathrm{E}_{i} / Z} \text { with } \quad Z=\sum_{i=0}^{N} e^{-\lambda \mathrm{E}_{i}}
$$

The absolutely identical solution is obtained when applying the variational procedure onto equation (54). This demonstrates that one of the most fundamental equations of thermodynamics can be derived from the mereological description as depicted in the present article. The constraints 
(Lagrange multipliers) being introduced into the original Gibbs's formulation to account for energy and probability conservation emerge fully naturally from the scheme being outlined in the present article.

\section{The contrast equation}

The basic equation can obviously be substracted from itself (just different indices are used):

$$
\sum_{\mathrm{i}=0}^{\mathrm{N}_{\Phi}} \Phi_{\mathrm{i}}-\sum_{\mathrm{j}=0}^{\mathrm{N}_{\Phi}} \Phi_{\mathrm{j}}=0
$$

In both cases the $\Phi_{\mathrm{i}}$ can - and shall - be arranged in a monotonously decreasing order with $\Phi_{0}$ being the largest value and $\Phi_{\mathrm{N}}$ being the smallest value. The equation can then be rewritten as

$$
\begin{gathered}
\left(\Phi_{\mathrm{N}_{\Phi}}+\sum_{\mathrm{i}=0}^{\mathrm{N}_{\Phi}-1} \Phi_{\mathrm{i}}\right)-\left(\Phi_{0}+\sum_{\mathrm{j}=1}^{\mathrm{N}_{\Phi}} \Phi_{\mathrm{j}}\right)=0 \\
\sum_{\mathrm{i}=0}^{\mathrm{N}_{\Phi}-1} \Phi_{\mathrm{i}}-\sum_{\mathrm{j}=1}^{\mathrm{N}_{\Phi}} \Phi_{\mathrm{j}}=\Phi_{0}-\Phi_{\mathrm{N}_{\Phi}}
\end{gathered}
$$

Renaming $j=i+1$ yields

$$
\begin{gathered}
\sum_{\mathrm{i}=0}^{\mathrm{N}_{\Phi}-1} \Phi_{\mathrm{i}}-\sum_{\mathrm{i}+1=1}^{\mathrm{N}_{\Phi}-1} \Phi_{\mathrm{i}+1}=\Phi_{0}-\Phi_{\mathrm{N}_{\Phi}} \\
\sum_{\mathrm{i}=0}^{\mathrm{N}_{\Phi}-1}\left(\Phi_{\mathrm{i}}-\Phi_{\mathrm{i}+1}\right)=\Phi_{0}-\Phi_{\mathrm{N}_{\Phi}} \\
\sum_{\mathrm{i}=0}^{\mathrm{N}_{\Phi}-1} \Delta \Phi_{\mathrm{i}}=\Phi_{0}-\Phi_{\mathrm{N}_{\Phi}} \\
\sum_{\mathrm{i}=0}^{\mathrm{N}_{\Phi}-1} \frac{\Delta \Phi_{\mathrm{i}}}{\Phi_{0}-\Phi_{\mathrm{N}_{\Phi}}}=1
\end{gathered}
$$

As the $\Phi_{\mathrm{i}}$ are monotonously decreasing, $\Phi_{0}$ and $\Phi_{\mathrm{N}}$ correspond to the maximum/minimum values $\Phi_{\max }$ and $\Phi_{\min }$, respectively, yielding the "contrast equation":

$$
\sum_{\mathrm{i}=0}^{\mathrm{N}_{\Phi}-1} \frac{\Delta \Phi_{\mathrm{i}}}{\Phi_{\max }-\Phi_{\min }}=1 \text { ("contrast equation") }
$$


The difference between the maximum and minimum values is the largest difference which can occur in the system

$$
\Phi_{\max }-\Phi_{\min }=\Delta \Phi_{\max }
$$

It never can reach the value of 1 because both, $\Phi_{\max }$ and $\Phi_{\min }$, are considered as existing and thus both have non-zero values :

$$
\Delta \Phi_{\max }<1
$$

The difference $\Delta \Phi_{\mathrm{i}}$ can be written as the change/variation of $\Phi$ with respect to some property multiplied with the difference in this property $x$

$$
\Delta \Phi_{\mathrm{i}}=\frac{\partial \Phi_{\mathrm{i}}}{\partial x} \Delta x
$$

Analogue for some other property $t$

$$
\Delta \Phi_{\mathrm{i}}=\frac{\partial \Phi_{\mathrm{i}}}{\partial t} \Delta t
$$

As an example, although neither space nor time have yet been introduced explicitly (but the basic equation holds anywhere and anytime, see above) $\frac{\partial \Phi_{i}}{\partial x}$ will be identified with the one dimensional spatial gradient - a scalar - represented by the Nabla symbol $(\nabla$, without arrow)

$$
\begin{gathered}
\Delta \Phi_{\mathrm{i}}=\nabla \Phi_{\mathrm{i}} \Delta x \\
\Delta \Phi_{\max }=\nabla \Phi_{\max } \Delta x \\
1>\Delta \Phi_{\max }=\nabla \Phi_{\max } \Delta x
\end{gathered}
$$

In a limiting case the maximum contrast asymptotically approaches the value of 1

$$
1 \cong \nabla \Phi_{\max } \Delta x
$$

Accordingly, there is a characteristic length corresponding to the steepest gradient which may asymptotically be reached in the system. This length is called $l_{p}$ as it probably is related to the Planck length:

$$
\begin{array}{r}
\Delta x=\frac{1}{\nabla \Phi_{\max }}=l_{p} \\
(\text { Planck length })
\end{array}
$$

In a similar way the maximum contrast in time can be exploited, which introduces the Planck time as related to the maximum change rate

$$
\begin{gathered}
\Delta t=\frac{1}{\frac{\partial \Phi_{\max }}{\partial t}}=\tau_{p} \\
(\text { Planck time })
\end{gathered}
$$

Inserting the gradient expressions (66) into the contrast equation (61) yields

$$
\sum_{\mathrm{i}=0}^{\mathrm{N}_{\Phi}-1} \frac{\nabla \Phi_{\mathrm{i}} \Delta x}{\nabla \Phi_{\max } \Delta x}=1
$$

and eventually 


$$
\sum_{\mathrm{i}=0}^{\mathrm{N}_{\Phi}-1} \nabla \Phi_{\mathrm{i}} \mathrm{l}_{\mathrm{p}}=1
$$

The same operations performed on the "basic equation" which eventually yielded the entropy equation (see preceding chapters; equations (14) through (18)) allow to derive a "gradient entropy" expression to be derived from equation (71):

$$
S=-\sum_{\substack{i=0 \\ \text { ("gradient entropy equation") }}}^{N_{\Phi^{-1}}}\left(\nabla \Phi_{\mathrm{i}} l_{p}\right) \ln \left(\nabla \Phi_{\mathrm{i}} l_{p}\right)
$$

This term (and its generalization to scalar products comprising 3 dimensional gradients) - being derived from other concepts at that time [31] - was used in previous articles by the author

(i) to derive the phase-field equation from an entropic approach [31].

(ii) to derive the "entropy of a geometric sphere" which has been shown to have the identical structure of the Bekenstein-Hawking entropy of a black hole [24],[32]:

$$
\begin{gathered}
S_{B H} \sim \frac{A}{4 l_{p}^{2}} \\
\text { ("black hole entropy") }
\end{gathered}
$$

(iii) to derive a formula describing gravity. The derived formula comprises a combination of terms similar-at least in a qualitative way-to terms appearing in a number of other theories of gravitation. In its simplest approximation the derived equation recovers the classical Newtonian law [33]:

$$
\begin{gathered}
\Delta \Phi=4 \pi \mathrm{G} \rho_{\mathrm{i}} \\
(\text { "Newton }- \text { Poisson equation") }
\end{gathered}
$$

The full equation especially comprises terms related to curvature of space and terms corresponding to so-called modified Newtonian dynamics "MOND" [34] being one of the concepts not drawing on the existence of dark matter to explain e.g. velocity distributions in galaxies.

\section{Outlook}

In this article the "basic equation" has been introduced. This equation - and its square - have then been multiplied with a scalar real value. This scalar has been shown to be possibly composed of other scalars and only the combined scalar has been shown to be a conserved quantity.

Besides scalars, a number of other mathematical entities relevant to physics can be distinguished as disjoint classes according to their behavior under symmetry operations like e.g. mirroring:

\begin{tabular}{|l|l|l|l|}
\hline \multicolumn{1}{|c|}{ entity } & \multicolumn{1}{|c|}{ example } & \multicolumn{1}{c|}{$\begin{array}{c}\text { behavior under } \\
\text { mirroring }\end{array}$} & \multicolumn{1}{c|}{ remarks } \\
\hline scalar & $\begin{array}{l}\text { energy, } \\
\text { time }\end{array}$ & invariant & $\begin{array}{l}\text { scalars are scalar products of two vectors or } \\
\text { two pseudovectors }\end{array}$ \\
\hline
\end{tabular}




\begin{tabular}{|c|c|c|c|}
\hline $\begin{array}{l}\text { vector/ } \\
\text { polar vector }\end{array}$ & $\begin{array}{l}\text { position, } \\
\text { momentum }\end{array}$ & change of sign & \\
\hline $\begin{array}{l}\text { pseudovector/ } \\
\text { axial vector }\end{array}$ & spin, torque & invariant & $\begin{array}{l}\text { pseudovectors typically are vector products of } \\
\text { (polar) vectors }\end{array}$ \\
\hline pseudoscalar & volume & change of helicity & $\begin{array}{l}\text { pseudoscalars are scalar products of vectors } \\
\text { and pseudovectors }\end{array}$ \\
\hline
\end{tabular}

Hypercomplex numbers have recently been shown to be capable of capturing all these types of mathematical entities in a single entity called octonion. Octonions have 8 real components: one for the scalar, three for the vector, three for the pseudovector and one for the pseudoscalar [35].

Octonions and their algebra are an extension of the quaternions. Quaternions were introduced by Hamilton in 1843. They were used by James Clark Maxwell to describe the Maxwell equations [36] before these were reformulated by Oliver Heaviside into the well-known vector calculus formulation [37]. Nowadays, "Hypercomplex numbers [38]-[40], especially quaternions, are widely used in relativistic mechanics, electrodynamics, quantum mechanics and quantum field theory [41]-[48] (see also the bibliographical review in [49])" (adapted from [35], [50])

It is beyond the scope of the present article to exploit anything what can be done when multiplying the basic equation with octonions or other complex/hypercomplex numbers. Multiplication of the basic equation with such numbers/entities can be expected to generate further insight and many further equations as compared to multiplication with scalars alone being depicted in the present article.

A short example is the multiplication with a vector $\vec{p}$ (e.g. momentum) or a pseudovector $\vec{L}$ (e.g. spin), which both are entities being disjoint from scalars and also disjoint amongst each other:

$$
\sum_{\mathrm{i}=0}^{\mathrm{N}_{\Phi}} \Phi_{\mathrm{i}} \vec{p}=\vec{p}=\sum_{\mathrm{i}=0}^{\mathrm{N}_{\Phi}} \overrightarrow{p_{l}} \quad \text { resp. } \quad \sum_{\mathrm{i}=0}^{\mathrm{N}_{\Phi}} \Phi_{\mathrm{i}} \vec{L}=\vec{L}=\sum_{\mathrm{i}=0}^{\mathrm{N}_{\Phi}} \overrightarrow{L_{l}}
$$

Taking the time derivative of these equations immediately indicates the respective vectors/pseudovectors to be further conserved quantities:

$$
\overrightarrow{0}=\sum_{\mathrm{i}=0}^{\mathrm{N}_{\Phi}} \dot{\vec{p}}_{l}
$$

("Conservation of momentum")

$$
\overrightarrow{0}=\sum_{\mathrm{i}=0}^{\mathrm{N}_{\Phi}} \overrightarrow{\vec{L}}_{l}
$$

\section{("Conservation of angular momentum")}

Another important, future extension of the scheme presented by now is seen in higher powers of the basic equation. Higher powers of the basic equation - besides binary correlations between pairs of things i. e. terms proportional to $\Phi_{i} \Phi_{j}$ - will lead to correlations between 3 and 4 things like $\Phi_{i} \Phi_{j} \Phi_{k}$ and $\Phi_{i} \Phi_{j} \Phi_{k} \Phi_{l}$. Such higher order correlations are related to the coexistence of three respectively four things. While two things coexist at boundaries (2D), three things can coexist at triple lines (1D) and four things only at quadruple points (0D).

\section{Conclusions}


Starting from the philosophical concept of mereology, reasoning for a very simple and seemingly very fundamental "basic equation" describing the sum of "things in a universe" has been provided. Especially this correlation of a philosophical principle with an algebraic equation makes the present article an "essay". All further operations on this basic equation, however, then are simple mathematical operations. In a deductive way the basic equation has been used to derive expressions for correlations between things which typically occur at interfaces. Terms describing interfaces have been shown to be related to correlations and to entropy and a number of physics equations has been derived from the same basic equation in the present article, Figure 3:

- Entropy equation

- Energy conservation equation

- Mass conservation equation

- Energy-Mass equivalence

- Lorentz factor

- Ideal gas law (with an important amendment)

- Boltzmann statistics

- Rydberg equation for the energy levels in hydrogen atoms

- vacuum energy

- a formulation for Planck length/time as the inverse of a maximum contrast gradient/transient

- the entropy of a sphere being identical to the black hole entropy [24]

- $\quad$ an equation for gravity [33]

- the phase-field equation [31]

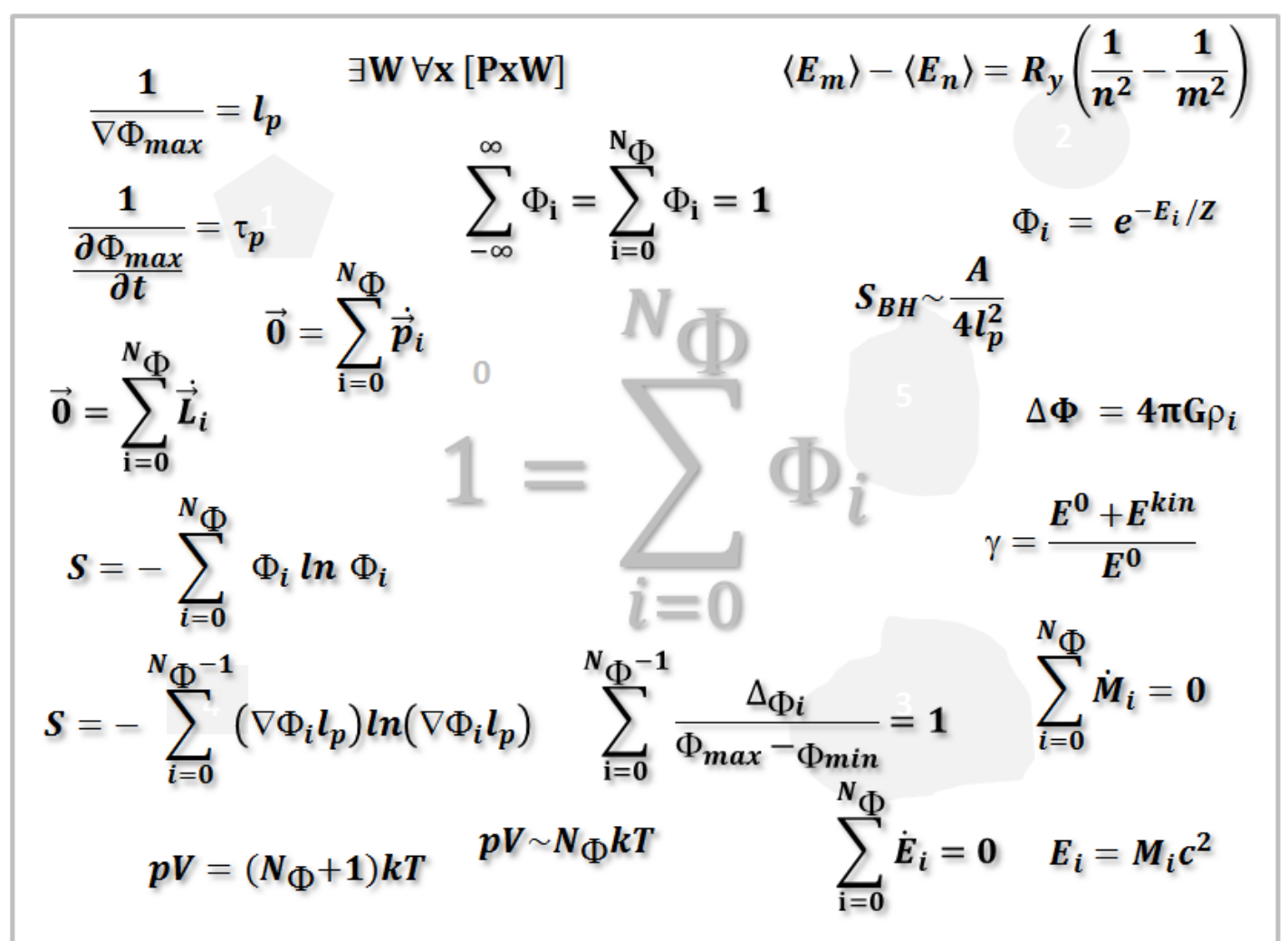

Figure 3: Graphical summary of the equations being derived from the basic equation (center). The "mereophysics" approach being detailed in the present article in a surprisingly direct way allowed deriving several well-known equations from physics by "quantifying" one of the axioms underlying the philosophical concept of mereology. Some of the equations shown were derived in previous articles and are based on the "gradient entropy" equation being depicted in the present 
article.

Concluding remark: This article is driven by personal enthusiasm of the author, who - after deriving the ideal gas equation from the mereological equation - was curious to see "what else" could be derived. In this sense it was the scope to derive "known equations" and these derivations themselves thus may be biased. Actually, however, the applied scheme unexpectedly has even led to a minor, but perhaps important, correction to the ideal gas equation. The consequences of this correction on the nature of vacuum energy have still to be further explored.

Funding: The work presented in this article is a spin-off from discussions during the formulation of a European Materials Modelling Ontology EMMO [6],[7] being funded by the European Commission in the EMMC-CSA and MarketPlace projects under grants \#723867 and \# 760173.

Acknowledgments: The author thanks Emanuele Ghedini, Jesper Friis, Gerhard Goldbeck, Adham Hashibon and Sebastiano Moruzzi for their patience and for all the interesting and constructive discussions on philosophy and on various areas of physics during the intense work on the European Materials \& Modelling Ontology.

Conflicts of Interest: The author declares no conflict of interest. 


\section{References}

1. https://en.wikipedia.org/wiki/Epistemology (accessed 26th of July 2019)

2. $\quad$ https://en.wikipedia.org/wiki/Gnosiology (accessed 26th of July 2019)

3. http://www.collinsdictionary.com/dictionary/english/gnosiology (accessed 26th of July 2019)

4. https://en.wikipedia.org/wiki/Ontology (accessed 26th of July 2019)

5. http://www.merriam-webster.com/dictionary/ontology (accessed 26th of July 2019)

6. E. Ghedini , G. Goldbeck, A. Hashibon, G. J. Schmitz, and J. Friis (2019): “Foundations of an ontology for materials and modelling", article submitted to "Universe" (July 2019)

7. EMMO: The European Materials \& Modelling ontology “EMMO" is freely available online at GitHub: https://github.com/emmo-repo/EMMO

8. https://en.wikipedia.org/wiki/Zermelo-Fraenkel_set_theory (accessed 26th of July 2019)

9. David K Lewis: "Parts of Classes" Wiley-Blackwell (1991) ISBN-13: 978-0631176565

10. https://en.wikipedia.org/wiki/Urelement (accessed 26th of July 2019)

11. https://en.wikipedia.org/wiki/Stanisław_Leśniewski (accessed 26th of July 2019)

12. Roberto Casati, Achille C. Varzi: Parts and Places: The Structures of Spatial Representation A Bradford Book; UK ed. (2003) ISBN-13: 978-0262517072

13. Franz Baader, Diego Calvanese, Deborah L McGuinness, Daniele Nardi, Peter F. Patel-Schneider. "The description logic handbook: theory, implementation, and applications". Cambridge University Press New York, NY, USA. 2003. ISBN:0-521-78176-0.

14. https://en.wikipedia.org/wiki/Mereology (accessed 26th of July 2019)

15. I. Steinbach, F. Pezzolla, B. Nestler, M. Seeßelberg, R. Prieler, G.J. Schmitz, J.L.L. Rezende: „A phase field concept for multiphase systems" Physica D 94(1996), p.135-147

16. G.J. Schmitz, B. Böttger, J. Eiken, M. Apel, A. Viardin, A. Carré and G. Laschet: "Phase-field based simulation of microstructure evolution in technical alloy grades" Int. J. Adv. Eng. Sci. Appl. Math. 24 (2012) 126 DOI 10.1007/s12572-011-0026-y

17. George Boolos, "to be is to be the value of a variable (or to be some values of some variables)", Journal of Philosophy, 81(1984) , 430-49

18. E. Noether: Invariante Variationsprobleme. In: Gött. Nachr. 1918, S. 235-257. (see also: https://de.wikipedia.org/wiki/Noether-Theorem, accessed Feb 20th 2019)

19. Bronstein, I.N.; Mühlig, H.; Musiol, G.; Semendjajew, K.A. Taschenbuch der Mathematik (Bronstein); Deutsch, H., Ed.; Verlag: Harri Deutsch, Germany, 2016.

20. Willard, G.J. The Scientific Papers of J. Willard Gibbs; Ox Bow Press: Woodbridge, CT, USA, 1993; Vol. 1.

21. Willard, G.J. The Scientific Papers of J. Willard Gibbs; Ox Bow Press: Woodbridge, CT, USA, 1993; Vol 2.

22. Hasenöhrl, F. Wissenschaftliche Abhandlungen von Ludwig Boltzmann. I. Band (1865-1874) II. Band (1875-1881) and III. Band (1882-1905) Available online: https://phaidra.univie.ac.at/view/o:63647 (accessed on 22 May 2018). (In German).

23. Weaver, W.; Shannon, C.E. The Mathematical Theory of Communication; University of Illinois Press: Urbana, IL, USA, 1949; ISBN 0-252-72548-4.

24. Schmitz, G.J.: "Entropy and Geometric Objects", Entropy 2018, 20(6), 453; https://doi.org/10.3390/e20060453

25. Schmitz, G.J. et al.: Quantitative mereotopoplogy (under preparation)

26. https://de.wikipedia.org/wiki/Lorentzfaktor: Lorentz factor as function of kinetic energy

27. https://en.wikipedia.org/wiki/Cosmological_constant_problem (accessed 26th of July 2019)

28. Adler, Ronald J.; Casey, Brendan; Jacob, Ovid C.: "Vacuum catastrophe: An elementary exposition of the cosmological constant problem". American Journal of Physics. 63 (7): 620-626. doi:10.1119/1.17850.

29. https://en.wikipedia.org/wiki/Rydberg_constant (accessed 26th of July 2019)

30. E.T Jaynes: Probability Theory: The logic of Science, chapter 11, (internet: http://omega.albany.edu:8008/JaynesBook.html) (accessed March 2017)

31. G. J. Schmitz: “Thermodynamics of Diffuse Interfaces”, in H. Emmerich, B. Nestler, M. Schreckenberg (eds): "Interface and Transport Dynamics" Springer Lecture Notes in Computational Science and engineering, ISBN 3-540-40367-1 (2003) p. 47-64

32. Bekenstein, J.D. Black-hole thermodynamics. Phys. Today 1980, 33, 24-31. 
33. G. J. Schmitz: "A Combined Entropy/Phase-Field Approach to Gravity", Entropy 2017,19 (4),151; doi:10.3390/e19040151

34. Milgrom, M.: "A modification of the Newtonian dynamics as a possible alternative to the hidden mass hypothesis". Astrophys. J. Part 1 1983, 270, 365-370, doi:10.1086/161130.

35. V. L. Mironov and S. V. Mironov “Octonic Electrodynamics” https://arxiv.org/ftp/arxiv/papers/0802/0802.2435.pdf (accessed 26th of July 2019)

36. J. C. Maxwell, “A dynamical theory of the electromagnetic field”, Philosophical Transactions of the Royal Society of London,155(1865), 492

37. O. Heaviside, "Electromagnetic Theory", The Electrician Printing and Publishing Co., London, (1894).

38. Kantor I. L. and Solodovnikov A. S., Hypercomplex Numbers: an Elementary Introduction to Algebras (Springer-Verlag, 1989).

39. J. C. Baez, “The Octonions”, Bull. Amer. Math. Soc.,39(2001), 145

40. Okubo S., Introduction to Octonion and Other Non-associative Algebras in Physics (Montroll memorial lecture series in mathematical physics, 2), (Cambridge University Press, 1995).

41. Dixon G M., Division Algebras: Octonions, Quaternions, Complex Numbers and the Algebraic Design of Physics (Mathematics and its Applications), (Springer, 2006).

42. Smith D. and Conway J. H., On Quaternions and Octonions (Publisher AK Peters, 2003).

43. Adler S. L., Quaternionic Quantum Mechanics and Quantum Fields (New York: Oxford University Press, 1995).

44. Adler S. L., “Time-dependent perturbation theory for quaternionic quantum mechanics, with application to CP nonconservation in K-meson decays", Phys. Rev. D 34, 1871-1877 (1986).

45. Adler S. L., "Scattering and decay theory for quaternionic quantum mechanics, and the structure of induced T nonconservation", Phys. Rev. D 37, 3654-3662 (1988).

46. Davies A. J. and McKellar B. H. J., "Nonrelativistic quaternionic quantum mechanics in one dimension", Phys. Rev. A 40, 4209-4214 (1989).

47. Davies A. J., “Quaternionic Dirac equation”, Phys. Rev. D 41, 2628-2630 (1990).

48. De Leo S. and Rotelli P., “Quaternion scalar field”, Phys. Rev. D 45, 575-579 (1992).

49. Gsponer A. and Hurni J. P., “Quaternions in mathematical and physics (1)”, Preprint arXiv:math-ph/0510059 v3 (2006)

50. V. L. Mironov and S. V. Mironov, “Octonic representation of electromagnetic field equations", J. Math. Phys., 50(2009), 12901.

(C) 2019 by the authors. Submitted for possible open access publication under the terms and conditions of the Creative Commons Attribution (CC BY) license (http://creativecommons.org/licenses/by/4.0/). 